\title{
Compressed Sensing Soft Activity Processing for Sparse Multi-User Systems
}

\author{
Fabian Monsees, Carsten Bockelmann, Armin Dekorsy \\ Department of Communications Engineering \\ University of Bremen, Germany \\ E-mail: $\{$ monsees,bockelmann,dekorsy\}@ant.uni-bremen.de
}

\begin{abstract}
Performing joint activity and data detection has recently gained ubiquitous attention for reducing signaling overhead in multi-user Machine-to-Machine Communication systems. To this end investigations on the application of Compressed Sensing have focused on estimating data and node activity jointly in scenarios where the per node activity is very low. The focus of this paper is to enhance the performance of state of the art detectors by the utilization of soft information. In particular we provide a formulation of activity Log-Likelihood ratios, that we utilize to improve the activity detection.
\end{abstract}

\section{INTRODUCTION}

Machine Type Communication (MTC) is a fast growing field which constantly raises new demands on existing communication systems. Compared to human driven communication, MTC is quite often sporadic and of very low data rate making extensive signaling and complex scheduling inappropriate [1], [2]. Recent research has shown how signaling and scheduling complexity can be reduced by physical layer joint activity and data detection [3]-[5]. Physical layer joint activity and data detection assumes a multi-user uplink network, where nodes sporadically transmit data to a central aggregation node for further processing. Given some knowledge about the node activity, the detector blindly estimates the set of active nodes and the data. The reliability of the activity detection is crucial for the data-detection as erroneous activity detection strongly impacts the overall performance. Improving the performance at the activity detection is therefore a major problem in joint activity and data detection based systems.

This paper improves symbol-by-symbol joint activity and data detection [4] by utilizing soft information concerning the node activity. With attention to frame-wise processing we employ a scenario where nodes desire to transmit frames of data sporadically to a central aggregation node. Each node is assumed to be active only with a low probability, making the multi-user signal at the aggregation node sparse. In this setup we make use of the knowledge that nodes are either active or inactive for the duration of a whole frame. With this information we first extract activity Log-likelihood ratios (LLRs) indicating whether a node was more likely active or inactive. Second, we use these activity LLRs as a priori knowledge at the blind activity and data detector yielding a two stage detector. The investigations in this works combine

This work was funded by the German Research Foundation (DFG) under grant DE 759/3-1.
Compressed Sensing problems with the framework of soft information.

As we show at an exemplary simulation setup, our two stage detector outperforms symbol-by-symbol joint activity and data detection. Moreover, it can be shown that this two stage detection scheme performs very reliable, as the rate of nodes that is wrongly estimated to be active nearly vanishes. With the knowledge of a sparse multi-user signal, we generalize our scheme for under-determined systems where the number of variables exceeds the number of observations. As a major result, it can be shown that to some degree of under-determinedness the performance of this detector is nearly preserved.

\section{System Model}

We start by formulating a general Machine-to-Machine (M2M) uplink communication model where nodes desire to transmit a frame of data to a central aggregation node. The transmissions are assumed to be synchronized on frame level. More specifically the system consists of $K$ nodes that transmit a frame consisting of $L$ symbols to a central aggregation point. Motivated by smart-metering and industrial applications [6] each nodes transmits only occasionally and the probability that the $k$ th node starts at the beginning of a frame is $p_{a}$. If a node is active, it transmits a frame of $L$ consecutive data symbols modulated by a modulation alphabet $\mathcal{A}$, which can be, e.g., PSK. If a node is inactive the node does not access the medium and keeps silent for the duration of the whole frame. The detector models inactive nodes as transmitting zeros instead of modulation symbols and uses an augmented alphabet $\mathcal{A}_{0}=\mathcal{A} \cup\{0\}$ for detection.

This model interprets the set of nodes at any time instance as transmitting symbols from the augmented alphabet $\mathcal{A}_{0}$. Stacking the symbols at time instance $i, 1 \leq i \leq L$, results in the multi-user signal vector $\mathbf{x}_{i} \in \mathcal{A}_{0}^{K}$. Concatenating $L$ multiuser vectors into a frame yields the matrix $\mathbf{X} \in \mathcal{A}_{0}^{K \times L}$ where the $k$ th row $\mathbf{x}_{\bar{k}}$ contains $L$ consecutive symbols from node $k$. Without restricting to any specific communication scheme, we assume a transmission over a wireless channel that can be expressed via the linear input-output relation.

$$
\mathbf{Y}=\mathbf{T X}+\mathbf{N} \text {. }
$$

Here, $\mathbf{Y} \in \mathbb{R}^{M \times L}$ is the matrix of observations, $\mathbf{T} \in \mathbb{R}^{M \times K}$ summarizes the channels from the nodes to the aggregation 


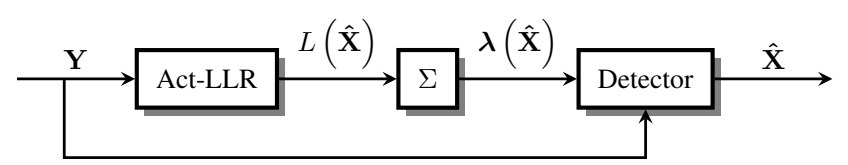

Fig. 1. Proposed two stage detector, which first estimates the set of active nodes and then estimates their data.

point and any type of medium access, e.g., Code division Multiple Access. The matrix $\mathbf{N} \in \mathbf{R}^{M \times L}$ contains i.i.d. zero-mean white Gaussian noise samples with variance $\sigma_{n}^{2}$ The previously introduced activity model allows to distinguish between two disjoint events modeling the node activity on a frame basis

$$
\begin{aligned}
& E 1: \mathbf{x}_{\bar{k}} \in \mathcal{A}^{L} \rightarrow \text { Node is active over frame } \\
& E 2: \mathbf{x}_{\bar{k}}=\mathbf{0}^{L} \rightarrow \text { Node is inactive over frame, }
\end{aligned}
$$

with probabilities $\operatorname{Pr}(E 1)=p_{a}$ and $\operatorname{Pr}(E 2)=1-p_{a}$. In the following we make use of the knowledge about the events $E 1$ and $E 2$ and extract soft information concerning the node activity from a frame of observations $\mathbf{Y}$.

\section{L-VALue BASED ACtivity Estimation}

\section{A. Overview of Frame Wise Processing}

Given a frame of observations, this section focuses on estimating the set of active nodes correctly. Previously, MAP detection has been employed on a symbol-by-symbol basis to jointly estimate activity and data [4], [7]. MAP detection could be extended for frame based systems which is due to the enormous complexity infeasible. However, we point out that symbol-by-symbol MAP detection fully neglects the fact that nodes are either active or inactive for the duration of a whole frame. Utilizing this knowledge can be used to improve the performance of joint activity and data detection.

In this paper we propose a two step detector outlined on Fig. 1 for separating activity and data detection. First, we calculate soft information concerning the node activity over a whole frame, thereby exploiting $E 1$ and $E 2$. Second, this knowledge is provided as a-priori information to a data detector, which performs symbol-by-symbol MAP detection over the frame.

The activity soft-information of the whole frame can be determined by first calculating activity LLRs for each time instance $i$ and then combining these for the whole frame. For each source symbol, the corresponding activity LLR gives an indication for the likelihood function $L\left(x_{k, i}\right)=\log \left(\operatorname{Pr}\left(x_{k, i}=0\right) / \operatorname{Pr}\left(x_{k, i} \in \mathcal{A}\right)\right)$. Given the activity LLRs for a whole frame $L(\mathbf{X})$, the node specific activity LLR can be obtained by summing up the activity LLRs for each node over a whole frame, i.e., summing over the columns in $L(\mathbf{X})$. This gives a reliable and robust per node activity LLR, which can be utilized as a-priori information for data detection.

\section{B. Activity Log-Likelihood Calculation}

With respect to the model given in (1), we now outline the processing steps for activity LLR estimation. The activity LLRs have to be calculated for each element $x_{k, i}$ via marginalization. In the following we denote the set of $K$ dimensional vectors with elements from the set $\mathcal{A}_{0}$ where the $k$ th element is equal to $\nu$ as $\mathbb{X}_{x_{k}=\nu}=\left\{\mathbf{x} \in \mathcal{A}_{0}^{K}: x_{k}=\nu\right\}$. Given a vector observation at time instance $i, \mathbf{y}_{i}=\mathbf{T} \mathbf{x}_{i}$ the activity LLR of the $k$ th node at this time instance can be cast as a Generalized Likelihood Ratio Test (GLRT) [8] between two marginalized distributions

$$
L\left(x_{k, i}\right)=\log \frac{\sum_{\mathbf{s} \in \mathbb{X}_{x_{k}=0}} p\left(\mathbf{y}_{i} \mid \mathbf{s}\right) \operatorname{Pr}(\mathbf{s})}{\sum_{\mathbf{s} \in \mathbb{X}_{x_{k} \in \mathcal{A}}} p\left(\mathbf{y}_{i} \mid \mathbf{s}\right) \operatorname{Pr}(\mathbf{s})} .
$$

With the formulation (3) we interpret the node activity as a binary hypothesis problem, i.e., node is active or inactive corresponding to a symbol-by-symbol interpretation of the events $E 1$ and $E 2$, respectively. Note, that the hypothesis for node activity constitutes a composite hypothesis involving all possible transmitted modulation symbols from the alphabet $\mathcal{A}$. With (1), the likelihood function $p\left(\mathbf{y}_{i} \mid \mathbf{s}\right)$ can be written as

$$
p\left(\mathbf{y}_{i} \mid \mathbf{s}\right) \propto \exp \left(-\frac{1}{2 \sigma_{n}^{2}}\left\|\mathbf{y}_{i}-\mathbf{T} \mathbf{s}\right\|_{2}^{2}\right) .
$$

Considering (4), we see that (3) evaluates a sum including $\mathcal{O}\left(\left|\mathcal{A}^{K}\right|\right)$ summands which leads to a high computational effort, especially if $K$ is large. The sum in (3) over exponential terms can be simplified by utilizing the so called max-log approximation [9] and thus turning the evaluation of the sum into an optimization problem yielding

$$
\begin{aligned}
L\left(x_{k, i}\right) & \approx \min _{\mathbf{s} \in \mathbb{X}_{x_{k} \in \mathcal{A}}}\left[\frac{1}{2 \sigma_{n}^{2}}\left\|\mathbf{y}_{i}-\mathbf{T} \mathbf{s}\right\|_{2}^{2}-\log (\operatorname{Pr}(\mathbf{s}))\right] \\
& -\min _{\mathbf{s} \in \mathbb{X}_{x_{k}=0}}\left[\frac{1}{2 \sigma_{n}^{2}}\left\|\mathbf{y}_{i}-\mathbf{T s}\right\|_{2}^{2}-\log (\operatorname{Pr}(\mathbf{s}))\right] .
\end{aligned}
$$

Using the activity model (2) and the corresponding event probabilities, the a-priori probability for the vector hypothesis $\mathbf{s} \in \mathcal{A}_{0}^{K}$ reads

$$
\begin{aligned}
& \log (\operatorname{Pr}(\mathbf{s}))=\log \left(\left(1-p_{a}\right)^{K-\|\mathbf{s}\|_{0}}\left(\frac{p_{a}}{|\mathcal{A}|}\right)^{\|\mathbf{s}\|_{0}}\right) \\
\Rightarrow & \log (\operatorname{Pr}(\mathbf{s})) \propto-\|\mathbf{s}\|_{0} \log \left(\frac{1-p_{a}}{p_{a} /|\mathcal{A}|}\right) .
\end{aligned}
$$

Here, $\|\mathbf{s}\|_{0}$ is the zero norm and simply counts the number of unequal zero elements contained in s [10]. Inserting (6) into (5) yields the max-log approximated activity LLR

$$
\begin{aligned}
& L\left(x_{k, i}\right) \approx \\
& \min _{\mathbf{s} \in \mathbb{X}_{x_{k} \in \mathcal{A}}}\left[\frac{1}{2 \sigma_{n}^{2}}\left\|\mathbf{y}_{i}-\mathbf{T s}\right\|_{2}^{2}+\|\mathbf{s}\|_{0} \log \left(\frac{1-p_{a}}{p_{a} /|\mathcal{A}|}\right)\right] \\
& -\min _{\mathbf{s} \in \mathbb{X}_{x_{k}=0}}\left[\frac{1}{2 \sigma_{n}^{2}}\left\|\mathbf{y}_{i}-\mathbf{T s}\right\|_{2}^{2}+\|\mathbf{s}\|_{0} \log \left(\frac{1-p_{a}}{p_{a} /|\mathcal{A}|}\right)\right] .
\end{aligned}
$$

The regularization term $\lambda=\log \left(\frac{1-p_{a}}{p_{a} /|\mathcal{A}|}\right)$ can be interpreted as a sparsity promoting term and the value of $\lambda$ determines 
how strong sparsity in $\mathbf{s}$ is promoted. It can observed that a convex relaxation of (7), i.e., replacing the zero norm by the $l_{1}$-norm and allowing continuous alphabets, leads to the so called least absolute shrinkage and selection operator (LASSO) which is commonly used for solving underdetermined Compressed Sensing problems [11] [12].

\section{Implementation via Modified Sphere Decoder}

Before describing the processing of the activity LLRs further, we briefly outline the calculation of the activity LLRs via a modified Sphere Decoding approach. Subsequently, it is shown how the second summand of (7) is solved via Sphere Decoding, the first summand is analogous and thus omitted. In the following we restrict ourselfs to a constant modulus modulation alphabet $\mathcal{A}$, i.e., $\left|x_{i}\right|=1 \forall x_{i} \in \mathcal{A}$ such as Phase Shift Keying (PSK). With this restriction, it is possible to replace the penalty term based on the zero norm by any norm and we have

$$
\lambda\|\mathbf{s}\|_{0}=\|\lambda \mathbf{s}\|_{1}=\|\sqrt{\lambda} \mathbf{s}\|_{2}^{2} \Leftrightarrow\left|s_{k}\right|=1 \forall k .
$$

This allows reformulating the optimization (7) problem to

$$
\begin{aligned}
& \min _{\mathbf{s} \in \mathbb{X}_{x_{k} \in \mathcal{A}}} \frac{1}{2 \sigma_{n}^{2}}\left\|\mathbf{y}_{i}-\mathbf{T s}\right\|_{2}^{2}+\|\mathbf{s}\|_{2}^{2}+[\lambda-1]\|\mathbf{s}\|_{0} \\
& \min _{\mathbf{s} \in \mathbb{X}_{x_{k} \in \mathcal{A}}} \frac{1}{2 \sigma_{n}^{2}}\left\|\left[\begin{array}{l}
\mathbf{y}_{i} \\
\mathbf{0}_{K}
\end{array}\right]-\left[\begin{array}{l}
\mathbf{T} \\
\mathbf{I}_{K}
\end{array}\right] \mathbf{s}\right\|_{2}^{2}+\underbrace{[\lambda-1]}_{\Theta}\|\mathbf{s}\|_{0}
\end{aligned}
$$

Sphere Decoding relies on an accumulating metric in the sequence of estimated elements. This can only be guaranteed if and only if $\Theta \geq 0$ holds. Without changing the optimization problem, (9) is rewritten such that the penalty term remains positive even if $\Theta<0$ holds. The key point is that we subtract the magnitude of $\Theta$ and add the constant $|\Theta| K$ to the problem. After re-arranging, $[K-\|\mathbf{s}\|]$ can be written as a sum of indicator functions $\mathbf{1}_{0}(\cdot)$, which returns a one if the argument is zero and a zero otherwise. For $\theta<0$ we obtain

$$
\begin{aligned}
& \min _{\mathbf{s} \in \mathbb{X}_{x_{k} \in \mathcal{A}}} \frac{1}{2 \sigma_{n}^{2}}\left\|\left[\begin{array}{l}
\mathbf{y}_{i} \\
\mathbf{0}_{K}
\end{array}\right]-\left[\begin{array}{l}
\mathbf{T} \\
\mathbf{I}_{K}
\end{array}\right] \mathbf{x}\right\|_{2}^{2}-|\Theta|\|\mathbf{s}\|_{0}+|\Theta| K \\
& \min _{\mathbf{s} \in \mathbb{X}_{x_{k} \in \mathcal{A}}} \frac{1}{2 \sigma_{n}^{2}}\left\|\left[\begin{array}{l}
\mathbf{y}_{i} \\
\mathbf{0}_{K}
\end{array}\right]-\left[\begin{array}{l}
\mathbf{T} \\
\mathbf{I}_{K}
\end{array}\right] \mathbf{x}\right\|_{2}^{2}+|\Theta|\left[K-\|\mathbf{s}\|_{0}\right] \\
& \min _{\mathbf{s} \in \mathbb{X}_{x_{k} \in \mathcal{A}}} \frac{1}{2 \sigma_{n}^{2}}\left\|\left[\begin{array}{l}
\mathbf{y}_{i} \\
\mathbf{0}_{K}
\end{array}\right]-\left[\begin{array}{l}
\mathbf{T} \\
\mathbf{I}_{K}
\end{array}\right] \mathbf{x}\right\|_{2}^{2}+|\Theta| \sum_{k=1}^{K} \mathbf{1}_{0}\left(x_{k}\right) \\
& \min _{\mathbf{s} \in \mathbb{X}_{x_{k} \in \mathcal{A}}} \frac{1}{2 \sigma_{n}^{2}}\left\|\mathbf{y}_{i}^{\prime}-\mathbf{T}^{\prime} \mathbf{s}\right\|_{2}^{2}+|\Theta| \sum_{k=1}^{K} \mathbf{1}_{0}\left(x_{k}\right),
\end{aligned}
$$

where the penalty term is always positive and can be summarized as

$$
f(\mathbf{s}, \Theta)=\left\{\begin{array}{l}
\sum_{k=1}^{K} \Theta \mathbf{1}_{\mathcal{A}}\left(s_{k}\right), \text { if } \Theta \geq 0, \\
\sum_{k=1}^{K}|\Theta| \mathbf{1}_{0}\left(s_{k}\right), \text { if } \Theta<0 .
\end{array}\right.
$$

Here, $f(\cdot)$ is a always positive function. Applying the skinny $\mathrm{QR}$ [13] decomposition on the augmented system description (10) results in $\mathbf{T}^{\prime}=\mathbf{Q R}$ with $\mathbf{Q} \in \mathbb{R}^{M+K \times K}$ being a matrix with orthonormal columns and $\mathbf{R} \in \mathbb{R}^{K \times K}$ being an upper triangular matrix. Filtering the received vectors $\tilde{\mathbf{y}}_{i}=\mathbf{Q y}_{i}$ leads in combination with the $\mathrm{QR}$ decomposition on (10) to

$$
\min _{\mathbf{s} \in \mathbb{X}_{x_{k} \in \mathcal{A}}} \frac{1}{2 \sigma_{n}^{2}}\left\|\tilde{\mathbf{y}}_{i}-\mathbf{R s}\right\|_{2}^{2}+f(\mathbf{s}, \Theta) .
$$

For the sake of space reasons we omit the algorithmic description of the utilized Sphere Decoder.

The key point is to consider solutions that lie within an ellipsoid around the received vector and adaptively decrement the search radius $d^{2}$ until the optimal value has been attained. The vector optimization problem (12) is thereby decomposed into $K$ coupled scalar optimization problems. This method can only succeed if each scalar optimization problem is strictly positive. Eq. (13) exemplary shows the scarlarized optimization problem (12).

$$
\begin{aligned}
& d^{2} \geq \frac{1}{2 \sigma_{n}^{2}}\left\|\tilde{\mathbf{y}}_{i}-\mathbf{R s}\right\|_{2}^{2}+f(\mathbf{s})= \\
& \frac{1}{2 \sigma_{n}^{2}} \sum_{u=1}^{K}\left(\tilde{y}_{i, u}-\sum_{v=1}^{K} r_{u, v} s_{v}\right)^{2}+f\left(s_{u}, \theta\right)
\end{aligned}
$$

With $f(\cdot ; \cdot)$ being always positive, allows for implementation via Sphere Decoding [14].

At this point it should be noted that even though Sphere Decoding is a quite efficient algorithm for solving the MAP problem, its runtime can only be bounded to be exponential on the dimensionality of the problem solved [15]. Besides Sphere Decoding, there exists a variety of sub-optimal algorithms that nearly achieve the performance of Sphere Decoding in polynomial time. The most prominent candidates are are Successive Interference Cancellation and K-Best detection [16].

\section{Activity LLR Processing and Data Detection}

Given a frame of observations $\mathbf{Y}$, the calculation of activity LLRs produces the matrix $L(\mathbf{X}) \in \mathbb{R}^{K \times L}$, where the $(k, i)$ th entry $L\left(x_{k, i}\right)$ denotes the activity LLRr of the the $k$ th node at time instance $i$ and corresponds to a solution of (5). Note that these LLRs only give an indicator for the activity of a node without giving any indication for the transmitted data itself. We decode the node specific activity pattern by summing over the rows of $L(\mathbf{X})$ yielding a vector $\boldsymbol{\lambda} \in \mathbb{R}^{K}$ whose entries correspond to the estimated frame-wise activity LLRs for the $K$ nodes over one frame. Here, the $k$ th element $\lambda_{k}$ corresponds to the activity LLR of the $k$ th node providing an estimate if the $k$ th node was more likely active or inactive for the duration of the considered frame. In particular the sign of the $k$ th element gives an estimator for $E 1$ or $E 2$ and the magnitude the reliability of the estimation.

As shown in Fig. 1 we provide the vector containing the node specific activity LLRs as a priori information to a symbol-by-symbol data detector [7]. The processing is as follows: the vector $\boldsymbol{\Theta}=\boldsymbol{\lambda}-\mathbf{1}_{K}$ is calculated and subsequently used in a Sphere Decoder as penalty term. Again, $f(\cdot ; \cdot)$ can 
be used to handle the regularization and we have to solve

$$
\hat{\mathbf{x}}_{i}=\arg \min _{\mathbf{x} \in \mathcal{A}_{0}} \frac{1}{2 \sigma_{n}^{2}}\left\|\tilde{\mathbf{y}}_{i}-\mathbf{R x}\right\|_{2}^{2}+\sum_{k=1}^{K} f\left(s_{k}, \Theta_{k}\right) .
$$

Solving (14) corresponds to the symbol-by-symbol MAP estimate for the data augmented by frame wise activity LLRs as a priori information. Note that (14) can be implemented via Sphere Decoding again. Solving (14) $L$ times gives the estimate for the complete data frame $\hat{\mathbf{X}}$ with taking the events $E 1$ and $E 2$ into regard.

\section{Simulative Results}

\section{A. Setup}

In the following, we investigate the performance for an exemplary multi-user uplink system. In particular, we investigate a Multi-User Code Division Multiple Access (CDMA) System, where a set of $K$ nodes accesses the wireless channel via CDMA transmitting data to a central aggregation node [17]. As stated in Section II, we assume a sporadic synchronous frame based medium access of the nodes parametrized by the per node activity probability $p_{a}$. In the following we further assume that the nodes are synchronous at chip level, which is not a general restriction as shown in [18], [19]. Moreover perfect Channel State Information is assumed at the aggregation node, which could, e.g., be obtained via a training phase at the beginning of each frame [20] or by random coding inspired techniques as introduced in [21]. Each node spreads its modulation symbols to a CDMA chip sequence of length $N$. These chips are transmitted over a frequency selective Rayleigh fading channel with length of $L_{h}$ chips. We model the input-output relation for this system by (1), where $\mathbf{T} \in \mathbb{R}^{N+L_{h}-1 \times K}$ summarizes spreading and convolution with the underlying frequency selective channel. To ensure fair comparisons, spreading sequences are normalized to have the same power such that the effective SNR does not depend on the spreading sequence length.

\section{B. Performance Analysis}

In the following, the performance is investigated in a fully loaded $N=K$ and overloaded $N<K$ CDMA setup. For comparison, we also apply symbol-by-symbol MAP detection as introduced in [7]. This detector simply estimates each source vector $\mathbf{x}_{i}$ based the activity probability $p_{a}$ without taking $E 1$ and $E 2$ into account and solves (14) $L$ times. The parameters of the simulation setup are summarized in Table I. As the presented detector mainly focuses on enhancing

\begin{tabular}{|c|c|}
\hline \multicolumn{2}{|c|}{ Simulation Parameters } \\
\hline \hline Number of Nodes & $K=20$ \\
\hline Frame-length & $L=100$ symbols \\
\hline Spreading Gain & $1 \leq N \leq 20$ \\
\hline Length of Channel Impulse Resp. & $L_{h}=4$ chips \\
\hline Channel Type & real valued block Rayleigh Fading \\
\hline Channel State Information & Perfect \\
\hline Activity Probability & $p_{a}=0.2$ \\
\hline Modulation Type & BPSK \\
\hline
\end{tabular}

TABLE I

SiMULATION PARAMETER

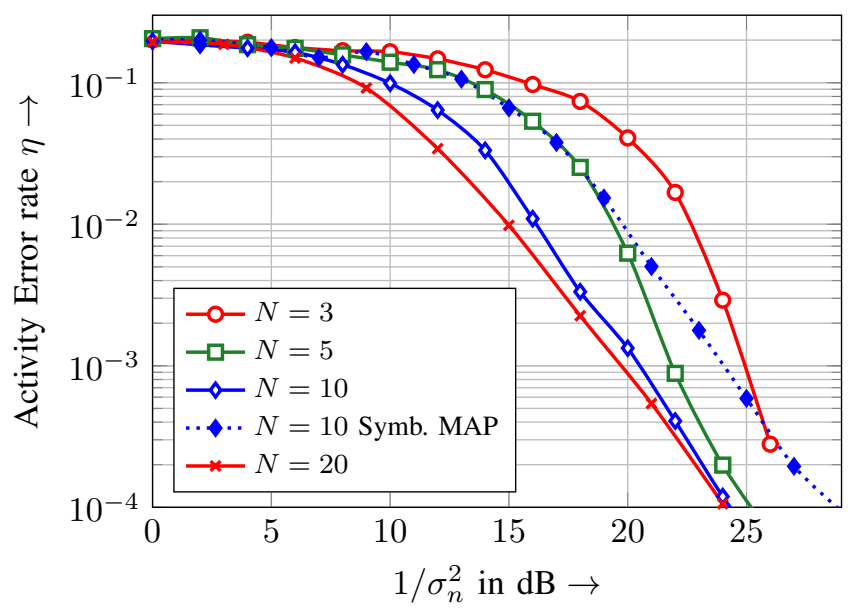

Fig. 2. Comparison of activity error rate of two step detector and symbolby-symbol MAP detector

the activity detection by calculating activity LLRs, we start by investigating the performance of the activity detection for different spreading sequence length $N$. The activity error rate is simply the summation of false alarm and missed detection errors yielding

$$
\eta=\mathrm{E}\left(\frac{|\{\hat{x} \in \mathcal{A}: x=0\}|+|\{\hat{x}=0: x \in \mathcal{A}\}|}{K L}\right) .
$$

Figure 2 shows the activity error rate $\eta$ for different length of spreading sequences. Decreasing the length from $N=20$ down to 10 obviously does not affect the system performance significantly. Even though the system is overloaded, the detector is still capable of correctly estimating the set of active nodes. Overloading the system further, $N=5$ and $N=3$ leads to losses regarding the activity detection. For comparison, Fig. 2 also shows the performance of a symbolby-symbol MAP detector [7]. The performance is exemplary shown for a system with a spreading sequence length of $N=10$. The gain of the novel two step detection is quite significant and accounts to approximately $5 \mathrm{~dB}$ performance gain compared to symbol-by-symbol MAP detection. Figure 2 shows the activity error rate which is composed of false alarm and missed detection errors. However, in our simulation we observed that the false alarm error rate is very low, i.e., $<10^{-3}$ for all our simulations. For practical considerations it is therefore justifiable to interpret the curves on 2 as missed detection rates.

In the following we investigate the impact of the frame length $L$ on the performance of the activity detection. The two step detector gains performance by utilizing the knowledge of frame-wise activity of the nodes in the system. Figure 3 shows the dependence of the activity error rate on the frame length $L$. The simulation parameters are the same as outlined in I. The spreading sequence length is chosen to $N=10$ and the SNR is $20 \mathrm{~dB}$. Most significantly, huge performance gains are already obtained for small frame lengths. A frame length of $L=20$ is nearly sufficient to significantly increase the performance of the two-step detector compared to symbol- 


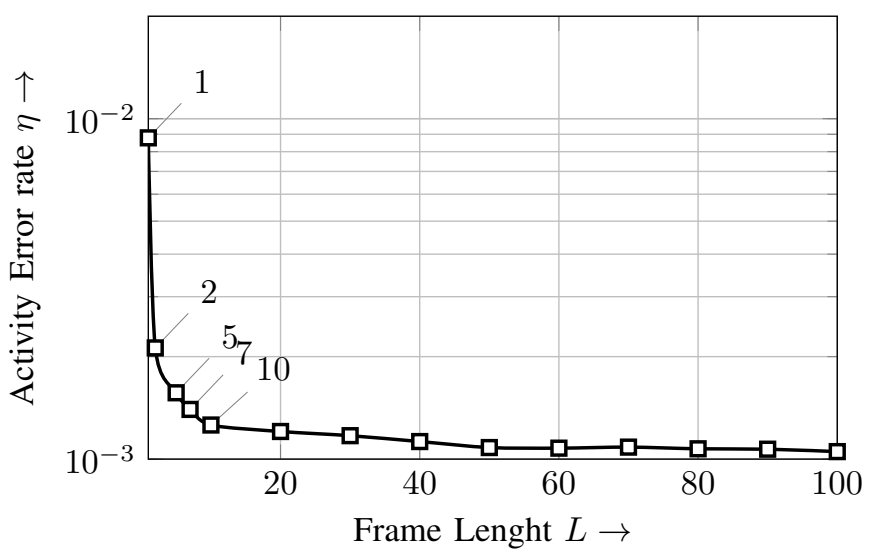

Fig. 3. Activity error rate over frame length $L$ with spreading sequence length $N=10$ and a fixed SNR of $20 \mathrm{~dB}$

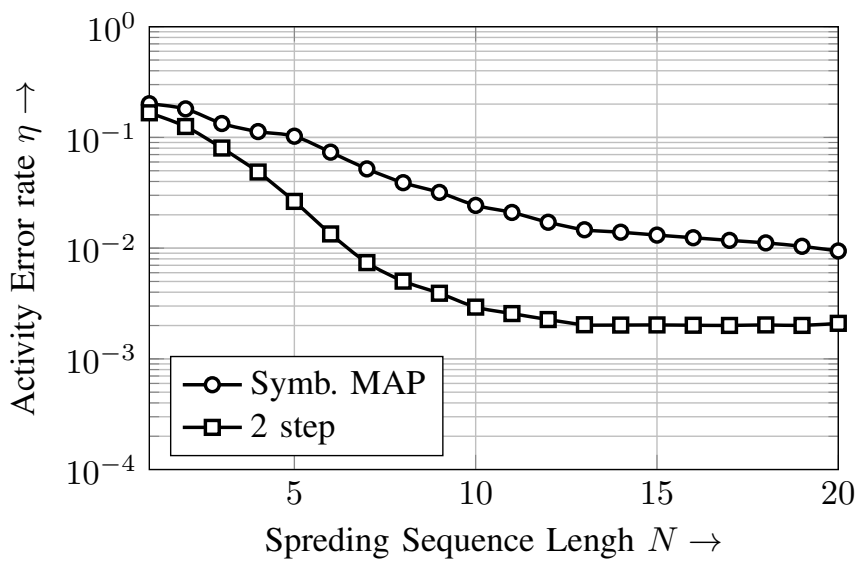

Fig. 4. Comparison of Activity Error rates at $\mathrm{SNR}=18 \mathrm{~dB}$ for varying spreading sequence length $N$

by-symbol MAP detection which does not utilize frame-wise node activity. Beyond frame lengths of $L=20$, gains are nearly negligible, showing that reliable activity detection can already be achieved with short frames of only a few symbols.

The performance gains of two step processing compared to symbol-by-symbol MAP over $N$ can be observed in Fig. 4. The error rates are shown at a fixed SNR $=18 \mathrm{~dB}$ while the spreading sequence length varies from $1 \leq N \leq 20$. Two step based detection outperforms symbol-by-symbol MAP detection for all simulated spreading sequence length $N$. Additionally, two step detection converges faster with a gain of nearly a decade at the SNR considered.

\section{CONCLUSION}

This paper has presented a detector that estimates user activity in a multi-user framework based on soft information about the frame activity of the users. We have presented a two step detector, where the first stage estimates the activity LogLikelihood ratios concerning the activity of the nodes in the system. In a second stage, symbol-by-symbol MAP detection is performed and the activity LLRs are utilized as a priori information at the detector. It could be shown that utilizing this type of prior information outperforms symbol-by-symbol MAP detection. Additionally it could be shown that most of the performance gains are obtained for small frame length already.

\section{REFERENCES}

[1] P. Jain, P. Hedman, and H. Zisimopoulos, "Machine type communications in 3gpp systems," IEEE Communications Magazine, vol. 50, no. 11, pp. 28-35, November 2012.

[2] V. Misic, J. Misic, and D. Nerandzic, "Extending LTE to support machine-type communications," in IEEE International Conference on Communications (ICC), 2012, Ottawa, Canada, June, pp. 6977-6981.

[3] H. Zhu and G. Giannakis, "Exploiting sparse user activity in multiuser detection," IEEE Transactions on Communications, vol. 59, no. 2, pp. 454 - 465, Feb 2011.

[4] F. Monsees, C. Bockelmann, D. Wübben, and A. Dekorsy, "Sparsity aware multiuser detection for machine to machine communication," in Second International Workshop on Machine-to-Machine Communication at IEEE Globecom 2012, Anaheim, USA, Dec 2012.

[5] H. F. Schepker and A. Dekorsy, "Sparse Multi-User Detection for CDMA transmission using greedy algorithms," in 8th International Symposium on Wireless Communication Systems (ISWCS), Aachen, Germany, Nov. 2011, pp. $291-295$.

[6] B. Andrea, M. Dohler, J. Hernndez-Serrano, A. Kountouris, and D. Barthel, "Low-Power Low-Rate goes Long-Range: the case for secure and cooperative Machine-to-Machine communications," in NETWORKING 2011 Workshops. Berlin, Heidelberg: Springer Berlin Heidelberg, 2011, vol. 6827, pp. 219-230.

[7] F. Monsees, C. Bockelmann, D. Wübben, and A. Dekorsy, "Compressed sensing bayes risk minimization for under-determined systems via sphere detection," in Accepted for publication in IEEE 77th Vehicular Technology Conference (VTC2013-Spring), Dresden, Germany, Jun 2013.

[8] A. S. Willsky and H. L. Jones, "A generalized likelihood ratio approach to state estimation in linear systems subjects to abrupt changes," in IEEE Conference on Decision and Control including the 13th Symposium on Adaptive Processes, vol. 13, Nov 1974, pp. 846 -853.

[9] C. Studer, M. Wenk, A. Burg, and H. Bolcskei, "Soft-output sphere decoding: Performance and implementation aspects," in Fortieth Asilomar Conference on Signals, Systems and Computers, 2006. ACSSC 2006. Pacific Groove, USA, Nov 2006, pp. 2071-2076.

[10] S. Boyd and L. Vandenberghe, Convex Optimization. Cambrigde University Press, 2004.

[11] R. Tibshirani, "Regression shrinkage and selection via the lasso," Journal of the Royal Statistical Society, Series B, vol. 58, no. 1, pp. 267-288, 1996.

[12] T. Park and G. Casella, "The bayesian lasso," Tech. Rep., 2005.

[13] G. H. Golub and C. F. V. Loan, Matrix Computations, 2nd ed. The Johns Hopkins University Press, 1989.

[14] M. O. Damen, H. E. Gamal, and G. Caire, "On maximum-likelihood detection and the search for the closest lattice point," IEEE Transactions on Information Theory, vol. 49, no. 10, pp. 2389 - 2402, Oct 2003.

[15] J. Jalden and B. Ottersten, "On the complexity of sphere decoding in digital communications," Signal Processing, IEEE Transactions on, vol. 53, no. 4, pp. 1474-1484, 2005.

[16] A. Burg, M. Borgmanr, M. Wenk, C. Studer, and H. Bolcskei, "Advanced receiver algorithms for mimo wireless communications," in Design, Automation and Test in Europe, 2006. DATE '06. Proceedings, vol. 1, 2006, pp. 6 pp.-.

[17] S. Verdú, Multiuser Detection. Cambridge, U.K.: Cambridge Univ. Press, November 1998.

[18] L. Applebaum, W. U. Bajwa, M. F. Duarte, and R. Calderbank, "Asynchronous code-division random access using convex optimization," Physical Communication, vol. 5, no. 2, pp. 129-147, Jun 2012.

[19] H. Schepker, C. Bockelmann, and A. Dekorsy, "Coping with CDMA asynchronicity in compressive sensing multi-user detection," in Accepted for publication at 2013 IEEE 77th Vehicular Technology Conference (VTC2013-Spring), Dresden, Germany, Jun 2013.

[20] - "Exploiting sparsity in channel and data estimation for sporadic multi-user communication," in 10th International Symposium on Wireless Communication Systems (ISWCS 13), Ilmenau, Germany, Aug 2013.

[21] M. Asif, W. Mantzel, and J. Romberg, "Channel protection: Random coding meets sparse channels," Oct 2009, pp. $348-352$. 